Pacific Journal of Mathematic 


\title{
SOME CONTENT MAXIMIZING PROPERTIES OF THE REGULAR SIMPLEX
}

\author{
R. Michael Tanner
}

In this paper it is shown that the regular simplex maximizes the sum of the squared contents of all $i$-dimensional faces, for all $i=2, \cdots, n$, when the sum of the one-dimensional squared contents is fixed. An immediate corollary is that the regular simplex has the largest total length of all joining lines, total area of all triangles, total volume of all tetrahedra, and so forth, for a fixed sum of squared line lengths. Some related unsolved conjectures are presented.

If a set of $n+1$ points in $n$-dimensional Euclidean space do not lie in an $(n-1)$-dimensional hyperplane, their convex hull is a simplex, the simplest of all $n$-dimensional polytopes. Any two points in the set determine a one-dimensional edge, or line, any three points determine a two-dimensional face, or triangle, and so forth. If all onedimensional edges have the same length, the simplex is called regular. If for no other reason than pure symmetry, the regular simplex should seem to be the extremum in any number of constrained maximization problems involving simplices. In this paper we consider a class of problems for which it is indeed maximal.

The author wishes to thank a conscientious referee who rewrote and substantially improved the exposition of the main result.

1. A symmetrization. Let $S$ be the simplex in $E^{n}$ with vertices $A_{1}, \cdots, A_{n+1}$ and let $V(i, j)=a_{i j}=\left|A_{i}-A_{j}\right|$ be a typical one-dimensional edge length. More generally, let $V\left(i_{1}, \cdots, i_{s}\right)$ be the content of the subsimplex formed by $A_{i_{1}}, \cdots, A_{i_{s}}$.

THEOREM. For $\sum a_{i j}^{2}>0$ fixed, the content power sum

$$
\sum V^{\lambda}\left(i_{1}, \cdots, i_{s}\right) \quad 0<\lambda \leqq 2,
$$

taken over all s-tuples $i_{1}<\cdots<i_{s}$, is maximal if and only if $S$ is a regular simplex.

The proof is based on a symmetrization which can be applied to any nonregular simplex to increase each of the sums while maintaining the constraint. We will give two equivalent constructions for the symmetrization: The first gives insight into its general nature, whereas the second is more immediately useful to the proof.

Given $A_{1}, \cdots, A_{n+1}$, let $J$ be the matrix whose $(i, j)$ th component is $A_{i} \cdot A_{j}$, a vector inner product. If the simplex is not regular, we 
can assume without loss of generality that $a_{i k} \neq a_{j k}$ for some $k$. Let $J_{i, j}$ be the matrix formed from $J$ by replacing every $(i, k)$ th component and $(k, i)$ th component by the $(j, k)$ th component and every $(j, k)$ th and $(k, j)$ th component by the $(i, k)$ th. In effect, this corresponds to an interchanging of the labels on $A_{i}$ and $A_{j}$. Then let $J^{\prime}=1 / 2\left(J+J_{i, j}\right)$. As the average of two nonnegative definite matrices, $J^{\prime}$ is itself nonnegative definite, so $J^{\prime}=P \Lambda P^{T}$, where $\Lambda=\left(\lambda_{i j}\right)$ is a diagonal matrix with nonnegative entries. Thus $J^{\prime}=$ $Q Q^{T}$ where $Q=P \Lambda^{1 / 2}$. The column vectors of $Q$ can be taken for $A_{1}^{\prime}, \cdots, A_{n+1}^{\prime}$, the vertices of a new simplex for which $\alpha_{i k}^{\prime}=a_{j k}^{\prime}$ for all $k$.

In physical terms, this construction is equivalent to rotating the $A_{i}-A_{j}$ edge into $E^{n+1}$, holding its midpoint fixed, until the edge is normal to the original $E^{n}$. The sum $\sum a_{i j}^{2}$ will not be affected, but the content power sum for $\lambda=2$ will become strictly larger unless the simplex is already regular. The reader might try this for $n=2$ to get the idea. To handle the general case, we always represent $E^{n}$ as the hyperplane $x_{n+1}=0$ in $E^{n+1}=\left\{\left(x_{1}, \cdots, x_{n}, x_{n+1}\right)\right\}$. Choose the origin at $1 / 2\left(A_{i}+A_{j}\right)$ and the $x_{1}$ axis so that $A_{i}=(1,0, \cdots, 0)$, and $A_{j}=(-1,0, \cdots, 0)$. Define $S^{\prime}=S(i, j ; x)$ to be the simplex formed from $S$ by replacing $A_{i}$ and $A_{j}$ with $A_{i}(x)=(x, 0, \cdots, 0$, $\left.\sqrt{\left(1-x^{2}\right)}\right)$ and $A_{j}(x)=\left(-x, 0, \cdots, 0, \sqrt{\left(1-x^{2}\right)}\right)$. Note that $A_{i}(1)=$ $A_{i}, A_{i}(-1)=A_{j}$, and similarly for $A_{j}$.

Lemma 1. If $A$ is any point in $E^{n}$ then

$$
\left|A_{i}(x)-A\right|^{2}=\left(\frac{1+x}{2}\right)\left|A_{i}-A\right|^{2}+\left(\frac{1-x}{2}\right)\left|A_{j}-A\right|^{2}
$$

and this remains true if $i$ and $j$ are interchanged; hence

$$
\left|A_{i}(x)-A\right|^{2}+\left|A_{j}(x)-A\right|^{2}=\left\{\left|A_{i}-A\right|^{2}+\left|A_{j}-A\right|^{2}\right\} .
$$

Proof. Simply write $A=\left(x_{1}, \cdots, x_{n}, 0\right)$ and calculate directly.

In what follows we call the translate of a subspace a flat, and write $d(K, p)$ for the distance from the set $K$ to the point $p$.

Lemma 2. Let $F$ be any flat of $E^{n}$. Then

$$
g(x)=d^{2}\left(F, A_{i}(x)\right)
$$

is concave in $x$.

Proof. By the Pythagorean theorem,

$$
d^{2}\left(F, A_{i}(x)\right)=1-x^{2}+d^{2}(F, p)
$$


where $p=(x, 0, \cdots, 0) \in E^{n}$. Clearly $d(F, p)=\left|\left(x-x_{0}\right) \sin \theta_{0}\right|$ for some real numbers $x_{0}$ and $\theta_{0}$, unless the $x_{1}$ axis is parallel to $F$, in which case it is constant. In either case $g(x)=a x^{2}+b x+c$ with $a \leqq 0$.

Note. The above remains true if $i$ is replaced by $j$. The function $g(x)$ is strictly concave unless the $x_{1}$-axis is normal to $F$.

Lemma 3. The distance from $(x, 0, \cdots, 0) \in E^{n+1}$ to a subspace of $E^{n}$ is at most the distance from $(0, \cdots, 0,1) \in E^{n+1}$ to that subspace for any $-1 \leqq x \leqq 1$.

Proof. The point $0 \in E^{n+1}$ belongs to the subspace.

Let $V\left(i, j, k_{1}, \cdots, k_{s} ; x\right)$ be the content of the subsimplex formed by $A_{i}(x), A_{j}(x), A_{k_{1}}, \cdots, A_{k_{s}}$.

Lemma 4. We have $V\left(i, j, k_{1}, \cdots, k_{s}\right) \leqq V\left(i, j, k_{1}, \cdots, k_{s} ; 0\right)$. Equality holds if and only if the line segment joining $A_{i}$ and the $A_{j}$ is normal to the flat $F$ determined by all the $A_{k_{m}}$ and the point $A_{0}=1 / 2\left(A_{i}+A_{j}\right)$.

Proof. The flat $F$ cuts the subsimplex into two (measure disjoint) simplices, each of which can be considered as having for its base the convex hull of $A_{0}$ and the $A_{k_{m}}$. The heights of these simplices are $d\left(F, A_{i}\right)$ and $d\left(F, A_{j}\right)$ respectively. By choosing coordinates as before we have $A_{0}=0$ and it follows from Lemma 3 with $x=1$ and $x=-1$ that $d\left(F, A_{i}(0)\right) \geqq d\left(F, A_{i}\right)$ and $d\left(F, A_{j}(0)\right) \geqq$ $d\left(F, A_{j}\right)$. The case of equality is obvious.

Lemma 5. If $A_{1}, \cdots, A_{k}$ are the vertices of a simplex and if the flat formed by any $k-2$ of them and the midpoint of the remaining two is normal to the line segment formed by the remaining two, then the simplex is regular.

Proof. Consider $A_{i}, A_{j}$, and $A_{k}$ where $i, j$ and $k$ are distinct. Since the flat determined by all vertices except $A_{j}$ and $A_{k}$ is equidistant from these two points, $d\left(A_{i}, A_{j}\right)=d\left(A_{i}, A_{k}\right)$. Since this is also true with $i$ and $j$ interchanged, every triangle is equilateral and the result follows.

We now prove the theorem. Consider the sum

$$
\sum(T)=\sum V^{2}\left(i_{1}, \cdots, i_{s}\right)
$$

both for $T=S$ and $T=S(i, j ; x)=S^{\prime}$. There are 3 types of terms: (i) those which involve neither $i$ nor $j$, (ii) those which involve exactly one of $i$ and $j$, and (iii) those which involve both $i$ and $j$. Terms 
of type (i) are the same for $S$ and $S^{\prime}$. By Lemma 4 terms of type (iii) for $S$ are at most as large as the corresponding terms for $S^{\prime \prime}$. We now group the terms of type (ii) in pairs so that the subscripts of two paired terms are the same except that one has an $i$ where the other has a $j$. Consider a typical pair. Let $B_{1}, \cdots, B_{r}$ be the vertices corresponding to their common subscripts, $H$ their convex hull, $V(H)$ its content, and $F$ the flat they determine. Then each pair is proportional to

$$
V^{2}(H) \cdot\left(d_{i}^{2}(x)+d_{j}^{2}(x)\right)
$$

where $d_{k}(x)=d\left(F, A_{k}(x)\right)$. Note that $d_{i}(1)=d_{j}(-1)$ and $d_{i}(-1)=d_{j}(1)$. By Lemma 2,

$$
\begin{aligned}
d_{i}^{2}(0)+d_{j}^{2}(0) & \geqq \frac{1}{2}\left(d_{i}^{2}(1)+d_{i}^{2}(-1)\right)+\frac{1}{2}\left(d_{j}^{2}(1)+d_{j}^{2}(-1)\right) \\
& =d_{i}^{2}(1)+d_{j}^{2}(1) .
\end{aligned}
$$

Thus $\sum(S) \leqq \sum(S(i, j ; 0))$. By Lemma 1 , when $S$ is replaced by $S(i, j ; 0)$ the quantity $\sum a_{i j}^{2}$ is not changed, and both distances $\left|A_{k}-A_{i}\right|$ and $\left|A_{k}-A_{j}\right|$, for any $k$, are replaced by their root mean square. Thus, by applying an infinite sequence of operations $S \rightarrow S(i, j ; 0)$ where every pair $(i, j)$ occurs infinitely often, one obtains a sequence of simplices $S=S_{1}, S_{2}, \ldots$ which converges to a regular simplex, and moreover $\sum\left(S_{1}\right) \leqq \sum\left(S_{2}\right) \leqq \cdots$. By Lemma 5 , one of these inequalities must be strict unless $S$ is regular.

Now for $0<\lambda \leqq 2$ the function $f(x)=x^{\lambda / 2}$ is concave, so if $N$ is the number of $V\left(i_{1}, \cdots, i_{s}\right)$ then

$$
\frac{1}{N} \sum f\left(V^{2}\left(i_{1}, \cdots, i_{s}\right)\right) \leqq f\left(\frac{1}{N} \sum V^{2}\left(i_{1}, \cdots, i_{s}\right)\right)
$$

i.e.

$$
\sum V^{\lambda}\left(i_{1}, \cdots, i_{s}\right) \leqq N^{1-\lambda / 2}\left(\sum V^{2}\left(i_{1}, \cdots, i_{s}\right)\right)^{\lambda / 2} .
$$

The right side is maximal if and only if $S$ is regular. However, if $S$ is regular then all terms in both sums are equal and we have equality. This completes the proof of the theorem.

COROLlary. Of all simplices whose vertices lie on a unit hypersphere $U$, the regular simplex uniquely maximizes the sum

$$
\sum V^{\lambda}\left(i_{1}, \cdots, i_{s}\right) \quad 0<\lambda \leqq 2
$$

taken over all s-tuples $i_{1}<\cdots<i_{s}$.

Proof. It is well known (see, e.g., [1]) that if $A_{1}, \cdots, A_{n+1}$ lie on $U$, then $\sum a_{i j}^{2}=(n+1)^{2}\left(1-d^{2}\right)$ where $d$ is the distance from the 
centroid of the vertices of the simplex to the center of the sphere. Since $d=0$ for the regular simplex, the result follows from the previous theorem.

2. Conjectures. The following conjectures concern the relationship between a hypersphere and a simplex. The interest in these particular questions comes from Information Theory: Proof of either would be sufficient to resolve the long-standing simplex code conjecture [2].

Let $S$ be an arbitrary simplex and $\hat{S}$ a regular simplex of $n$-dimensions centered on the origin. Define the function

$$
s(r)=\mu_{n-1}\{X \in S:|X|=r\}
$$

where $\mu_{n-1}$ is $(n=1)$-dimensional measure. The function thus represents the amount of a shell of radius $r$ contained within the simplex. $S$. Let $\hat{s}(r)$ be the corresponding function for the regular simplex. The first conjecture is that for a given intersection volume the regular simplex minimizes the shell, or surface, content.

Conjecture 1.

$$
\text { If } \int_{0}^{r_{0}} \hat{s}(r) d r=\int_{0}^{r_{0}} s(r) d r \text { then } \hat{s}\left(r_{0}\right) \leqq s\left(r_{0}\right) .
$$

The second conjecture is weaker than the first, being implied by the first. Now $S$ and $\hat{S}$ are restricted to being formed by hyperplanes which are all at some constant distance $d$ from the origin. Under the restriction, the conjecture is that $\widehat{S}$ minimizes the content in the following strict sense.

Conjecture 2.

$$
\hat{s}(r) \leqq s(r) \text { for all } r .
$$

Both conjectures seem intuitively correct, but the constraints are of a much greater complexity than those in the previous theorems.

3. Conclusions. The regular simplex is the obvious candidate for achieving the extremum of a number of functions defined on simplices under constraints. Intuitively its maximal symmetry must give it special properties. We have here explicitly shown for a set of problems a method for generating the symmetries in the course of the optimization; in addition we make some conjectures on other extremal properties. The symmetrization technique developed in this pursuit, which was originally motivated by a problem in communi- 
cation theory, appears to have potential in other areas related to group representation theory. Here we started with a matrix of vertex inner products and forced it, by a convexity operation, to be invariant under all permutations of row and column indices in the symmetric group of degree $n, S_{n}$. By restricting the group to be proper subgroup of $S_{n}$, a lower degree of symmetry would be imposed. This may then give a solution to extremal problems involving more complex polytopes.

\section{REFERENCES}

1. E. Hille, Some geometric extremal problems, J. Australian Math. Soc., 6 (1966), 122128.

2. R. M. Tanner, Contributions to the simplex code conjecture, Tech. Report No. 6151-8, Information Systems Lab, Stanford University, (1970).

Received August 8, 1973.

University of CAlifornia, SANTa Cruz 


\section{PACIFIC JOURNAL OF MATHEMATICS}

EDITORS

RICHARD ARens (Managing Editor)

University of California

Los Angeles, California 90024

\section{R. A. Beaumont}

University of Washington

Seattle. Washington 98105

\section{J. DugundjI}

Department of Mathematics University of Southern California Los Angeles, California 90007

D. Gilbarg and J. Milgram

Stanford University

Stanford. California 94305

\section{ASSOCIATE EDITORS}
E. F. BECKENBACH
B. H. NEUMANN
F. WOLF
K. Yoshida

\section{SUPPORTING INSTITUTIONS}

\author{
UNIVERSITY OF BRITISH COLUMBIA \\ CALIFORNIA INSTITUTE OF TECHNOLOGY \\ UNIVERSITY OF CALIFORNIA \\ MONTANA STATE UNIVERSITY \\ UNIVERSITY OF NEVADA \\ NEW MEXICO STATE UNIVERSITY \\ OREGON STATE UNIVERSITY \\ UNIVERSITY OF OREGON \\ OSAKA UNIVERSITY
}

\author{
UNIVERSITY OF SOUTHERN CALIFORNIA \\ STANFORD UNIVERSITY \\ UNIVERSITY OF TOKYO \\ UNIVERSITY OF UTAH \\ WASHINGTON STATE UNIVERSITY \\ UNIVERSITY OF WASHINGTON

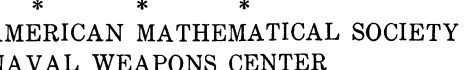

The Supporting Institutions listed above contribute to the cost of publication of this Journal, but they are not owners or publishers and have no responsibility for its content or policies.

Mathematical papers intended for publication in the Pacific Journal of Mathematics should be in typed form or offset-reproduced, (not dittoed), double spaced with large margins. Underline Greek letters in red, German in green, and script in blue. The first paragraph or two must be capable of being used separately as a synopsis of the entire paper. Items of the bibliography should not be cited there unless absolutely necessary, in which case they must be identified by author and Journal, rather than by item number. Manuscripts, in duplicate if possible, may be sent to any one of the four editors. Please classify according to the scheme of Math. Rev. Index to Vol. 39. All other communications to the editors should be addressed to the managing editor, or Elaine Barth, University of California, Los Angeles, California, 90024.

100 reprints are provided free for each article, only if page charges have been substantially paid Additional copies may be obtained at cost in multiples of 50 .

The Pacific of Journal Mathematics is issued monthly as of January 1966. Regular subscription rate: $\$ 72.00$ a year (6 Vols., 12 issues). Special rate: $\$ 36.00$ a year to individual members of supporting institutions.

Subscriptions, orders for back numbers, and changes of address should be sent to Pacific Journal of Mathematics, 103 Highland Boulevard, Berkeley, California, 94708.

\section{PUBLISHED BY PACIFIC JOURNAL OF MATHEMATICS, A NON-PROFIT CORPORATION}

Printed at Kokusai Bunken Insatsusha (International Academic Printing Co., Ltd.), 270, 3-chome Totsuka-cho. Shinjuku-ku, Tokyo 160. Japan.

Copyright (C) 1973 by Pacific Journal of Mathematics Manufactured and first issued in Japan 


\section{Pacific Journal of Mathematics}

\section{Vol. 52, No. $2 \quad$ February, 1974}

Harm Bart, Spectral properties of locally holomorphic vector-valued functions .....

J. Adrian (John) Bondy and Robert Louis Hemminger, Reconstructing infinite

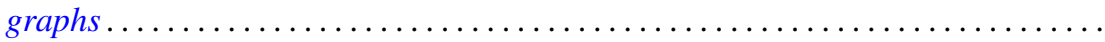

Bryan Edmund Cain and Richard J. Tondra, Biholomorphic approximation of planar

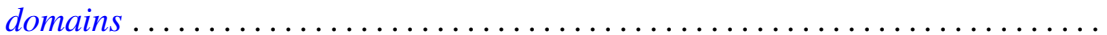

Richard Carey and Joel David Pincus, Eigenvalues of seminormal operators,

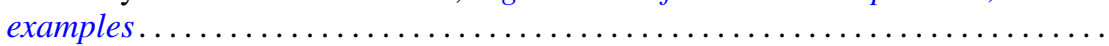

Tyrone Duncan, Absolute continuity for abstract Wiener spaces . . . . . . . . . . . . Joe Wayne Fisher and Louis Halle Rowen, An embedding of semiprime

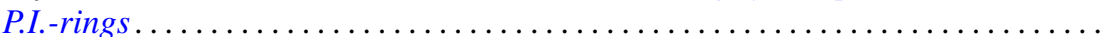

Andrew S. Geue, Precompact and collectively semi-precompact sets of semi-precompact continuous linear operators. . . . . . . . . . . . . . . .

Charles Lemuel Hagopian, Locally homeomorphic $\lambda$ connected plane continua ..... . Darald Joe Hartfiel, A study of convex sets of stochastic matrices induced by

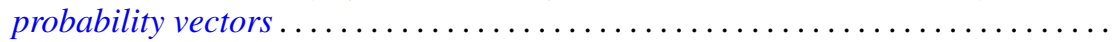

Yasunori Ishibashi, Some remarks on high order derivations $\ldots \ldots \ldots \ldots \ldots \ldots \ldots$ Donald Gordon James, Orthogonal groups of dyadic unimodular quadratic forms.

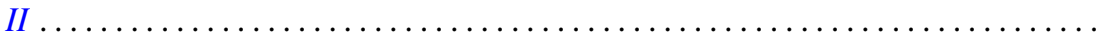

Geoffrey Thomas Jones, Projective pseudo-complemented semilattices . . . . . . . . . Darrell Conley Kent, Kelly Denis McKennon, G. Richardson and M. Schroder,

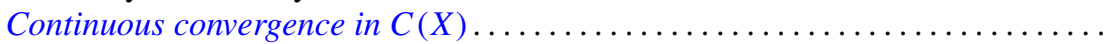

J. J. Koliha, Some convergence theorems in Banach algebras ...

Tsang Hai Kuo, Projections in the spaces of bounded linear oper

George Berry Leeman, Jr., A local estimate for typically real functions . .

475

Andrew Guy Markoe, A characterization of normal analytic spaces by the

homological codimension of the structure sheaf .........

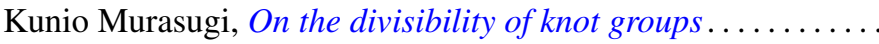

John Phillips, Perturbations of type I von Neumann algebras.

Billy E. Rhoades, Commutants of some quasi-Hausdorff matrices . .

David W. Roeder, Category theory applied to Pontryagin duality

Maxwell Alexander Rosenlicht, The nonminimality of the differential closure .

Peter Michael Rosenthal, On an inversion theorem for the general Mehler-Fock transform pair.

Alan Saleski, Stopping times for Bernoulli automorphisms

John Herman Scheuneman, Fundamental groups of compact complete locally affine complex surfaces. II. ........................

Vashishtha Narayan Singh, Reproducing kernels and operators with a cyclic vector. I. .

Peggy Strait, On the maximum and minimum of partial sums of random variables.

J. L. Brenner, Maximal ideals in the near ring of polynomials modulo 2 .

Ernst Gabor Straus, Remark on the preceding paper: "Ideals in near rings of polynomials over a field" ..........................

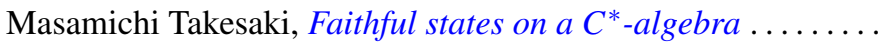

R. Michael Tanner, Some content maximizing properties of the regular simplex.

Andrew Bao-hwa Wang, An analogue of the Paley-Wiener theorem for certain

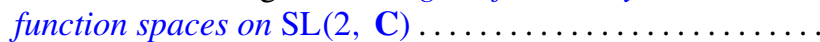

